\title{
Intestinal accumulation of silica particles in a rat model of dextran sulfate sodium-induced colitis
}

\author{
Shu Yie Janine Tamª, Janet K. Coller ${ }^{b}$, Anthony Wignalla,c, Rachel J. Gibson ${ }^{\mathrm{d}, \mathrm{e}}$, Aparajita Khatrif, \\ Chris Barbéf, Joanne M. Bowena \\ Adelaide Medical School, University of Adelaide; University of South Australia, Adelaide, South Australia, Australia
}

Abstract

aiscipline of Physiology, Adelaide Medical School, University of Adelaide, Adelaide, South Australia, Australia (Shu Yie Janine Tam, Anthony Wignall, Joanne M. Bowen); ' Discipline of Pharmacology, Adelaide Medical School, University of Adelaide, Adelaide, South Australia, Australia (Janet K. Coller); 'School of Pharmacy and Medical Sciences, University of South Australia, Adelaide, South Australia, Australia (Anthony Wignall); ${ }^{\mathrm{d} D i s c i p l i n e ~ o f ~ A n a t o m y, ~ A d e l a i d e ~ M e d i c a l ~ S c h o o l, ~ U n i v e r s i t y ~ o f ~ A d e l a i d e, ~}$ Adelaide, South Australia, Australia (Rachel J. Gibson); ${ }^{\mathrm{e} D i v i s i o n}$ of Health Sciences, University of South Australia, Adelaide, South Australia, Australia (Rachel J. Gibson); ${ }^{\mathrm{f} C e r a m i s p h e r e ~ P t y ~ L t d, ~ C o l l e g e ~ S t r e e t ~ G l a d e s v i l l e, ~ N e w ~}$ South Wales (Aparajita Khatri, Chris Barbé), Australia

This work was a collaborative endeavor between the University of Adelaide and Ceramisphere Pty Ltd.

Conflict of Interest: None

Correspondence to: Shu Yie Janine Tam, Department of Physiology, Adelaide Medical School, University of Adelaide, Adelaide, South Australia 5005, Australia, e-mail: shuyiejanine.tam@adelaide.edu.au

Received 30 April 2019; accepted 18 July 2019;

published online 3 August 2019

DOI: https://doi.org/10.20524/aog.2019.0411

\section{Introduction}

Ulcerative colitis (UC) is a chronic and lifelong condition characterized by continuous inflammation of the colonic mucosa; it has an incidence of 11.2 per 100,000 Australians [1]. The etiology of UC is still unconfirmed. However, the currently accepted hypothesis is the unsuppressed activation of both the body's innate and adaptive immunity due to unsuitable immune responses to the luminal antigens (bacteria, yeast) in the intestines [2]. Sustained epithelial damage caused by the imbalance of anti-inflammatory (interleukin- 4 and tumor necrosis factor $[\mathrm{TNF}]-\alpha)$ and pro-inflammatory (interleukin-1 and interferon- $\gamma$ ) cytokines, leads to an increase in membrane permeability and bacterial infiltration, triggering inflammation [3,4]. The damage manifests as diarrhea due to the influx of water into the lumen of the colon caused by the increase in membrane permeability, while the tissue damage causes abdominal pain, rectal bleeding, and anemia [4]. 
The approach to managing UC is to decrease colonic inflammation and induce remission of the disease. A range of approaches are clinically available, matching the severity of the disease and inflammatory state. Infliximab, a monoclonal antibody targeting TNF- $\alpha$, is currently being used to treat moderate to severe UC [5]. Since biological agents are unstable, infliximab needs to be administered intravenously (every 8 weeks) to avoid the acidic and enzyme-rich environments of the upper digestive tract [6]. Although effective at dampening inflammation, this method of administering therapy is associated with an increased (50\%) risk of infections, procedural failures and clinical medication errors [6,7]. Other UC treatments available, such as prednisolone, are administered orally. They are partially digested in the stomach before reaching the affected site in the colon, thereby reducing efficacy [8]. To overcome efficacy flaws in orally administered treatments, patients are required to take multiple doses a day or are prescribed higher doses, generally recognized to cause more severe adverse effects compared to single dose treatments $[9,10]$. UC treatments requiring intravenous or rectal (e.g., 5-aminosalicylate) administration are burdensome to patients [6,11]. While colectomies and proctocolectomies are last-resort treatments, they are invasive and, as such, are associated with more complications [6]. Given the substantial drawbacks of the delivery methods of current treatments, an alternative method of drug delivery for the treatment of UC is warranted.

Encapsulation in silica particles may overcome the challenges of oral delivery of fragile bioactive agents [12,13]. Silica is an FDAapproved material because of its stable and inert structure, which maximizes safety and treatment efficiency [14]. Silica particles are also biocompatible and will not induce any undesired toxic, immunogenic or carcinogenic responses [14]. They will also be degraded into orthosilicic acid, a water-soluble compound that humans naturally absorb, thus avoiding issues related to tissue accumulation in long-term use [14]. Silica particles comprise a silica matrix produced under physiologically relevant conditions, making them well-suited to biological therapies [12]. For example, a recent study by Zhang et al has shown poly(lactic-co-glycolic acid) nanoparticles loaded with a ginger active compound were successful in targeting colitis tissue in mouse models [15]. While silica particles have been shown to survive the gastrointestinal tract (GIT) environment [16], it is unknown whether these unloaded silica particles will preferentially accumulate and be retained at the sites of inflammation in the distal colon. The current study therefore aimed, firstly, to investigate the accumulation of unloaded, non-functionalized silica particles in a dextran sulfate sodium (DSS)-induced UC rat model and, secondly, to determine if there was a correlation between the accumulation of silica particles and colonic inflammation.

\section{Materials and methods}

\section{Silica particles}

Silica particles were synthesized at room temperature using Ceramisphere's proprietary technology, which employs a combination of emulsion and sol-gel technology to form matrix particles $[13,17]$. For these experiments, the silica particle surface was non-functionalized, without any functional groups. The fluorescent dye Alexa-633 was incorporated during the synthesis and was part of the silica matrix.

\section{DSS model of UC}

The study was approved by the University of Adelaide Animal Ethics Committee (M-2016-153) and complied with the National Health and Research Council Australia Code of Practice for Animal Care in Research and Training (2013) [18]. Male albino Wistar rats weighing between 200 and $250 \mathrm{~g}$ (6-7 weeks of age) were obtained from the Animal Resource Centre (Perth, Australia) and were group housed in ventilated cages ( $n=4-6$ rats/cage) with a 12-h light/dark cycle, and autoclaved food and water provided ad libitum.

Rats received either $4.5 \%$ DSS $(n=6)$ in drinking water or normal water $(\mathrm{n}=6)$ for 7 days. On day $7,10 \%$ sucrose solution replaced all drinking water to encourage rehydration. On day 9, Alexa Fluor (AF633)-tagged silica particles (200-300 nm diameter) were orally gavaged $(100 \mathrm{mg} / \mathrm{kg})$ into both DSS and control rats after $12 \mathrm{~h}$ of fasting to minimize any autofluorescence signal related to ingested rat chow. The silica particle size was chosen in order to avoid any issues related to particle toxicity [19]. Rats were live imaged at 3, 6, and $24 \mathrm{~h}$ after the oral gavage and killed on day 10.

\section{Live animal imaging of fluorescence}

Rats were anesthetized using isoflurane ( $2 \%$ in oxygen, flow rate $1.5 \mathrm{~L} / \mathrm{min}$ ) and their abdomen, from diaphragm to hips, was shaved before the movement of the silica particles was visualized using a biophotonic imaging system, Lumina IVIS XRMS using Living Imaging 3.2 (PerkinElmer, Massachusetts, United States) at 3,6, and $24 \mathrm{~h}$ after gavage of silica particles. Parameters used during live imaging were as follows: excitation and emission wavelength 620 and $670 \mathrm{~nm}$, respectively; exposure time auto; f/stop 2; binning 4; and field of view $12.5 \mathrm{~cm}$. Quantification of fluorescence from the live images was performed using the same software, by creating a region of interest within the shaved area. Fluorescent activity of the silica particles was measured as radiant efficiency: $\left.\left[\mathrm{p} / \mathrm{s} / \mathrm{cm}^{2} / \mathrm{sr}\right] /[\mu \mathrm{W}) / \mathrm{cm}^{2}\right]$, where $\mathrm{p}=$ pixel, $\mathrm{s}=$ second, $\mathrm{cm}^{2}=$ area, $\mathrm{sr}=$ steradian, and $\mu \mathrm{W}=$ microwatt.

\section{Assessment of UC disease activity}

Rats were weighed daily and checked twice/day for the presence of weight loss, diarrhea, and bleeding. The disease activity index (DAI) was quantified as the total of scores from 0-4 for the following parameters, where a higher score indicates more severe disease [20]:

- weight loss: $0 \%=0 ; 1-5 \%=1 ; 5-10 \%=2 ; 10-20 \%=3$; $>20 \%=4$ 
- $\quad$ stool consistency: normal stools, well-formed pellets $=0$; pasty stools, soft stools not stuck to the anus $=2$; diarrhea, liquid stools stuck to the anus $=4$

- perianal bleeding: no bleeding $=0$; occult, bleeding not visible $=2$; frank, visible bleeding $=4$.

\section{Tissue collection and preparation}

At the endpoint of the experiment, under deep inhalation anesthetic using isoflurane ( $2 \%$ in oxygen, flow rate $1.5 \mathrm{~L} / \mathrm{min}$ ), all rats were killed via cardiac exsanguination, followed by cervical dislocation. The entire GIT was removed and the cecum, small and large intestines were flushed with chilled 1 $\times$ phosphate buffered saline $(5 \mathrm{~mL})$ to remove the contents, collected into sterile containers. The small intestines and colons of all rats underwent IVIS imaging before being divided to be fixed in $10 \%$ neutral buffered formalin for $24 \mathrm{~h}$, transferred to $70 \%$ ethanol and embedded in paraffin wax, or immediately snap-frozen in liquid nitrogen and stored at $-80^{\circ} \mathrm{C}$ for further analysis.

\section{Tissue analysis}

All rats underwent tissue analysis. Hematoxylin and eosin (H\&E) staining was performed using $4 \mu \mathrm{m}$ sections of the jejunum, ileum, proximal (33\% length) and distal (33\% length) colon, cut on a rotary microtome and mounted onto glass microscope slides. Slides were scanned and assessed $(100 \times$ magnification) using an Olympus BX51 microscope (Olympus Corporation, Tokyo, Japan).

All slides underwent qualitative histopathological assessment to generate an injury score [21]. The histological criteria used in the assessment were as follows: epithelial disruption, crypt loss, crypt abscesses, goblet cell loss, submucosal thickening, muscularis externa thickening, and polynuclear cell infiltration [21]. Each parameter was scored as: 0 = absent, $1=$ mild, $2=$ moderate, $3=$ most severe, with a possible maximum score of 21 .

Staining with 4,6-diamidino-2-phenylindole (DAPI) was also performed on $4 \mu \mathrm{m}$ sections of the jejunum, ileum, proximal and distal colon mounted and coverslipped using Fluoroshield $^{\mathrm{TM}}$ from Sigma Aldrich (NSW, Australia). Slides were visualized using the Olympus FV3000 confocal laser scanning microscope (Olympus Corporation, Tokyo, Japan) to determine the percentage area of accumulation of the silica particles. Fluorescent images from the DAPI and AF633-silica particles were captured using different laser wavelengths (461 and $647 \mathrm{~nm}$, respectively) and the resulting high-resolution images taken at $400 \mathrm{x}$ magnification with a scan size of 1024 $\mathrm{x} 1024 \mathrm{~cm}$ were overlaid. The percentage area of fluorescence from the AF633-silica particles was quantified using ImageJ software V1.6.0 (National Institutes of Health, Maryland, United States) [22].

\section{Statistical analysis}

Data were compared using Prism version 7.0 (GraphPad ${ }^{\circledast}$ Software, San Diego, USA). Two-way analysis of variance (ANOVA) with Sidak's multiple comparisons test was performed to identify statistically significant differences between groups in DAI and radiant efficiency over time. For pairwise comparisons of non-parametric data, a MannWhitney $U$ test was performed. Correlations between data sets were evaluated using Spearman's rank correlation coefficient $\left(r_{s}\right)$. A P-value of $<0.05$ was considered significant.

\section{Results}

\section{DSS model of UC}

The DSS model of UC was characterized by determining the time course of damage in the distal colon of rats. Rats began to display features of UC, characterized by loose stool consistency, on day 3 after DSS was initiated, with occult bleeding becoming evident before progressing to frank bleeding at day 5 and to peak DAI at day 7 (Fig. 1A). DSS rats displayed a significant reduction in weight gain compared to controls from day 2 (Fig. 1B), and stool consistency and perianal bleeding scores of DSS rats peaked at day 7 (Fig. 1 C,D).

\section{Histopathological analysis of distal colon}

H\&E images of the distal colon after 7 days of DSS consumption showed mild histopathological injury compared to control rats (Fig. 2 A,B), as evidenced by epithelial disruption, neutrophil infiltration and submucosal thickening. Quantitative histological assessment of the injury showed a significant difference in scores after 7 days of DSS administration (Fig. 2C): control rats had a median score of 1.5 compared to 4 for DSS rats $(\mathrm{P}=0.0087)$. The colons of DSS rats were significantly shorter compared to control rats (Fig. 2D): control rats had a median length of $20.5 \mathrm{~cm}$ compared to $18.5 \mathrm{~cm}$ for DSS rats $(\mathrm{P}=0.0087)$.

\section{Live animal (in vivo) and ex vivo imaging of fluorescence}

Three hours after the oral administration of silica particles, the majority of the fluorescence signal was densely accumulated in the stomach in rats from both groups (Fig. $3 \mathrm{~A}, \mathrm{~B}$ ). At $6 \mathrm{~h}$, silica particle accumulation was more diffuse in rats from both groups. This was mirrored by the lower mean radiant efficiency at $6 \mathrm{~h}$ compared to $3 \mathrm{~h}$. By $24 \mathrm{~h}$, silica particle accumulation was completely diffuse from the GIT in rats from both groups; however, a high fluorescence signal was seen at the esophagus and perianal region of the rats. The ex vivo images (Fig. 3 C,D), of the GIT of rats from both groups showed a lower accumulation of silica particles at the distal colon. In the analysis of the live 

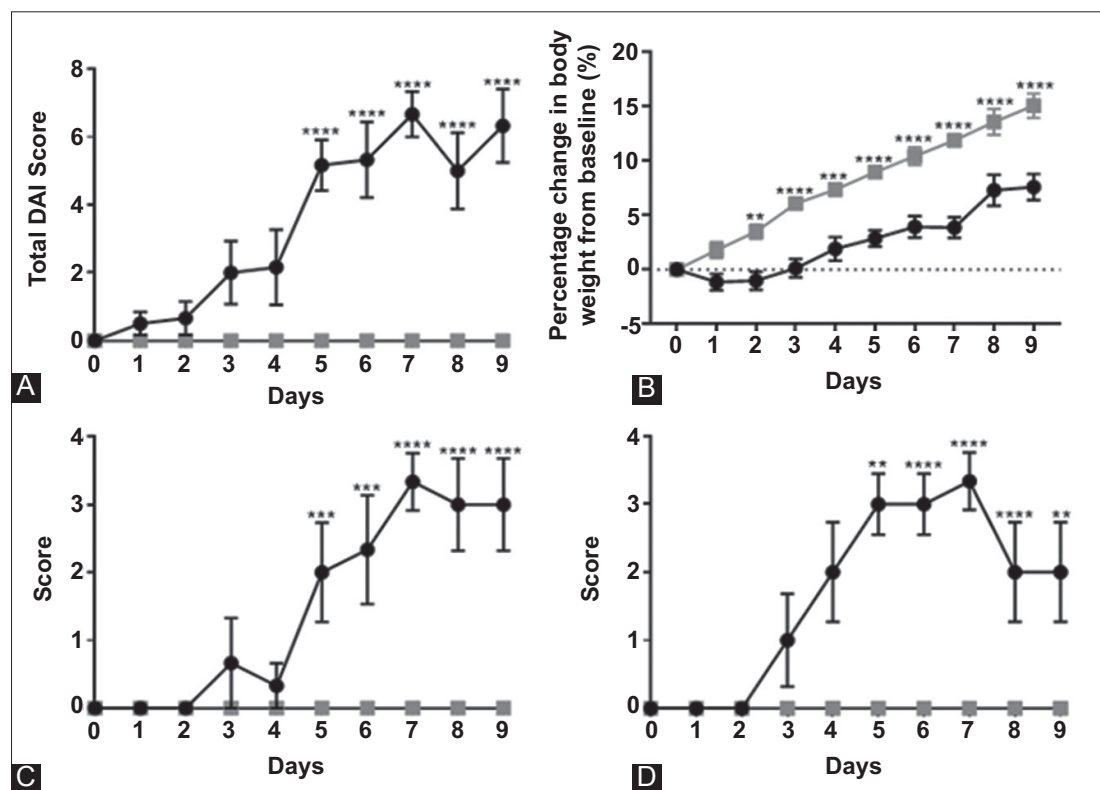

Figure 1 Time course (A) of disease activity index (DAI) in ulcerative colitis (UC), (B) changes in body weight from baseline, (C) stool consistency, and (D) perianal bleeding in rats treated with dextran sulfate sodium (DSS) compared with controls

Data are shown as mean \pm standard error of the mean (SEM). ${ }^{* *} P<0.01$, ${ }^{* * *} P<0.001$, ${ }^{* * *} P<0.0001$ versus control

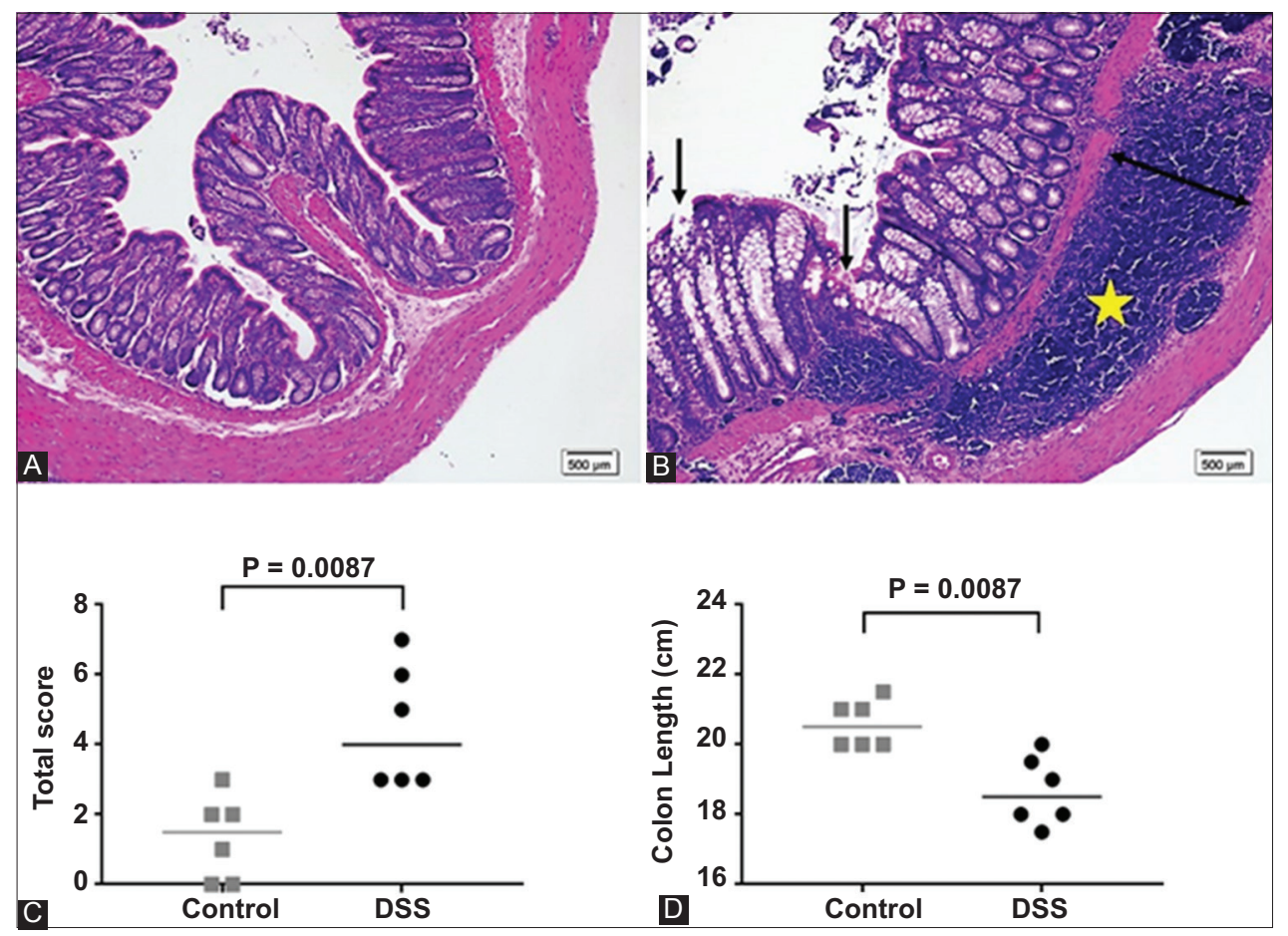

Figure 2 Representative hematoxylin and eosin staining (100× magnification) of distal colon of rats following 7 days of (A) water, and (B) $4.5 \%$ dextran sulfate sodium (DSS) solution. Symbols in (B) indicate epithelial disruption ( $\uparrow$ ), neutrophil infiltration (yellow star) and submucosal thickening $(\uparrow)$. (C) Histopathological scores and (D) colon lengths compared between DSS and water treated groups. Lines represents medians

animal (in vivo) images, quantification of fluorescence revealed no significant differences in silica particle accumulation between groups at 3,6, and $24 \mathrm{~h}$ after gavage (Fig. 4A). In addition, no significant difference in the signal intensities of the GIT images was observed between the groups (Fig. 4 B-E).

\section{Ex vivo fluorescence in GIT tissue}

Silica particles accumulated in the superficial aspect of the mucosa in the GIT (Fig. 5 A,B). There were no significant differences between the DSS and control groups in the 
percentage area of fluorescence in the fields of view of the GIT (Fig. 5 C-F).

\section{Correlation between histopathological injury, colon length, and silica particle accumulation in the colon}

There were no significant correlations between the histopathological scores of the rat distal colon and the radiant efficiency of the colon or the percentage area of fluorescence $\left(r_{s}=0.27\right.$ and -0.18 , respectively) (Fig. 6 A,B). Furthermore, there were no significant correlations between the colon length and the radiant efficiency of the colon or the percentage area of fluorescence $\left(r_{s}=-0.28\right.$ and 0.23 , respectively) (Fig. 6 C,D).

\section{Discussion}

Conventional colon-targeted methods of therapy, such as intravenous therapy and rectal administration, are currently used for the treatment of UC [23]. However, these delivery methods are associated with various disadvantages, such as greater infection rates and poor quality of life for patients [23]. Improved methods are needed for the oral delivery of therapeutics for the management of UC, especially when current treatment delivery methods are associated with these risks. New orally administered therapies that specifically target the colon form an active area of research for treating inflammatory bowel diseases, especially UC. The present study therefore aimed to investigate the potential of mesoporous silica particles as an alternative method of therapy delivery in a rat model of UC. Key findings indicated that the silica particles are able to travel to the distal colon. However, there was no significant difference in silica particle accumulation between a healthy and an inflamed colon, suggesting that more sophisticated targeting is needed if silica particles are to be effective carriers.

The DSS rat model was chosen for its similarity to clinical UC, its reproducibility and ease of administration [24]. DSS is a known anticoagulant as well as a chemical colitogen [25]

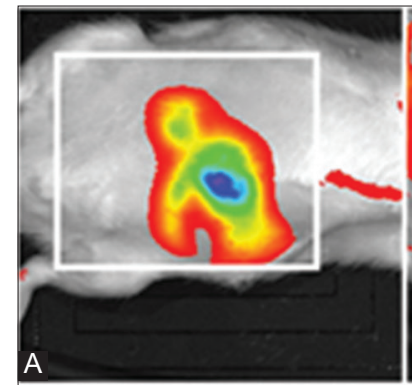

3 Hours
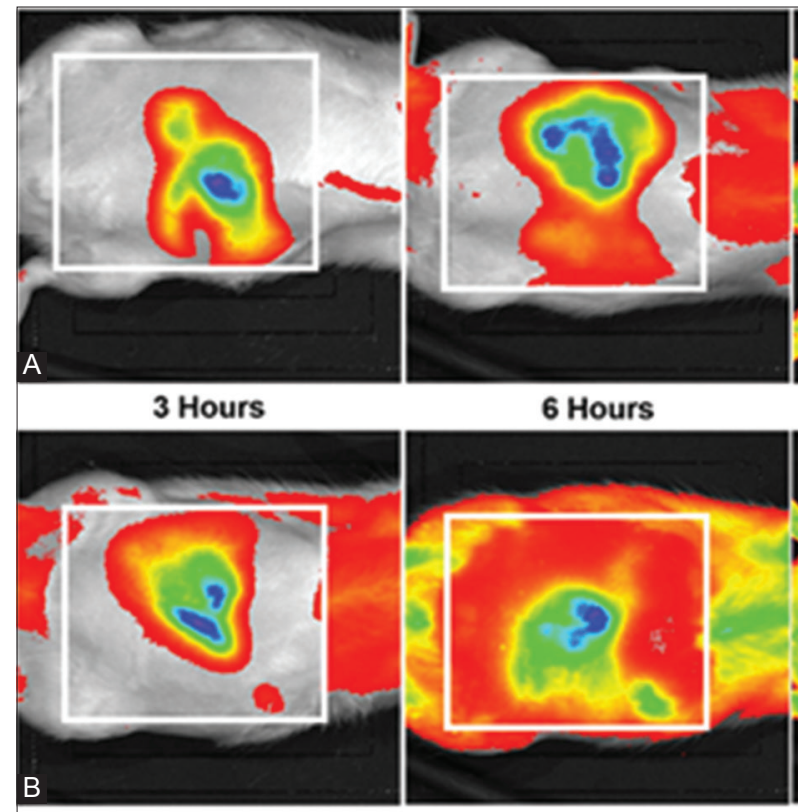

6 Hours
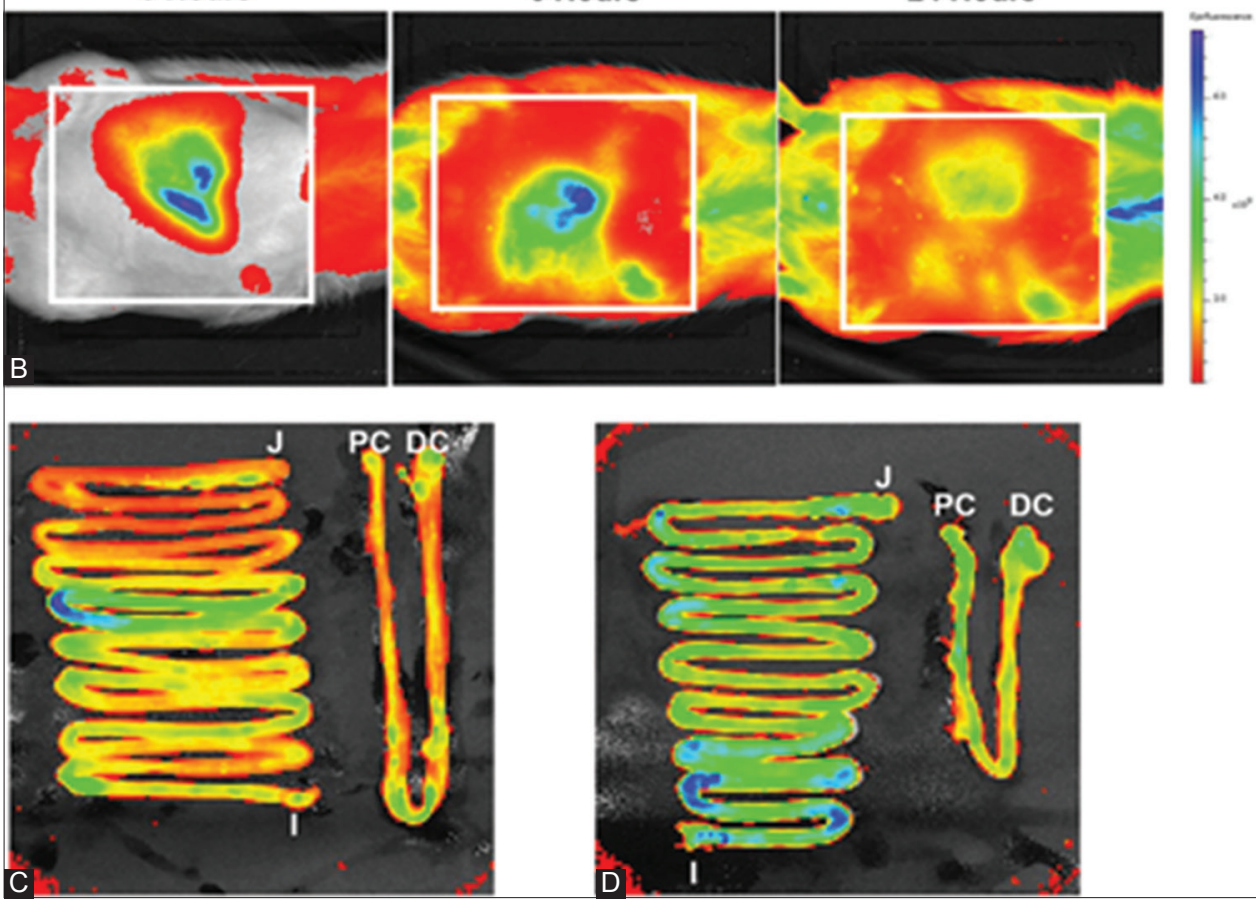
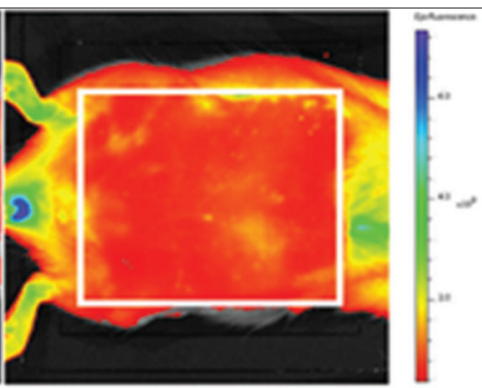

24 Hours

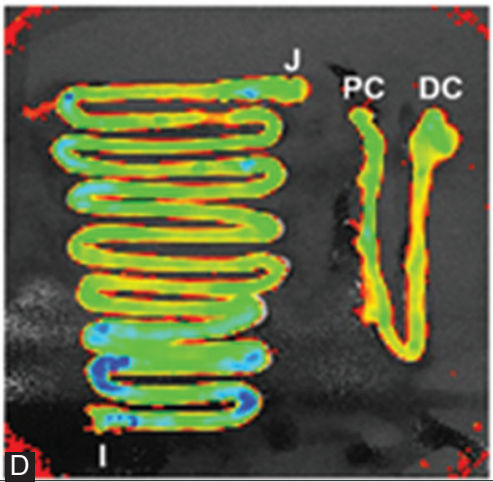

Figure 3 Representative in vivo optical images at 3,6, and 24 h post-gavage of silica particles in (A) control and (B) dextran sulfate sodium (DSS)-treated rats. Representative fluorescent intestinal tract images of (C) control and (D) DSS rats. Blue: high fluorescence, red: low fluorescence 


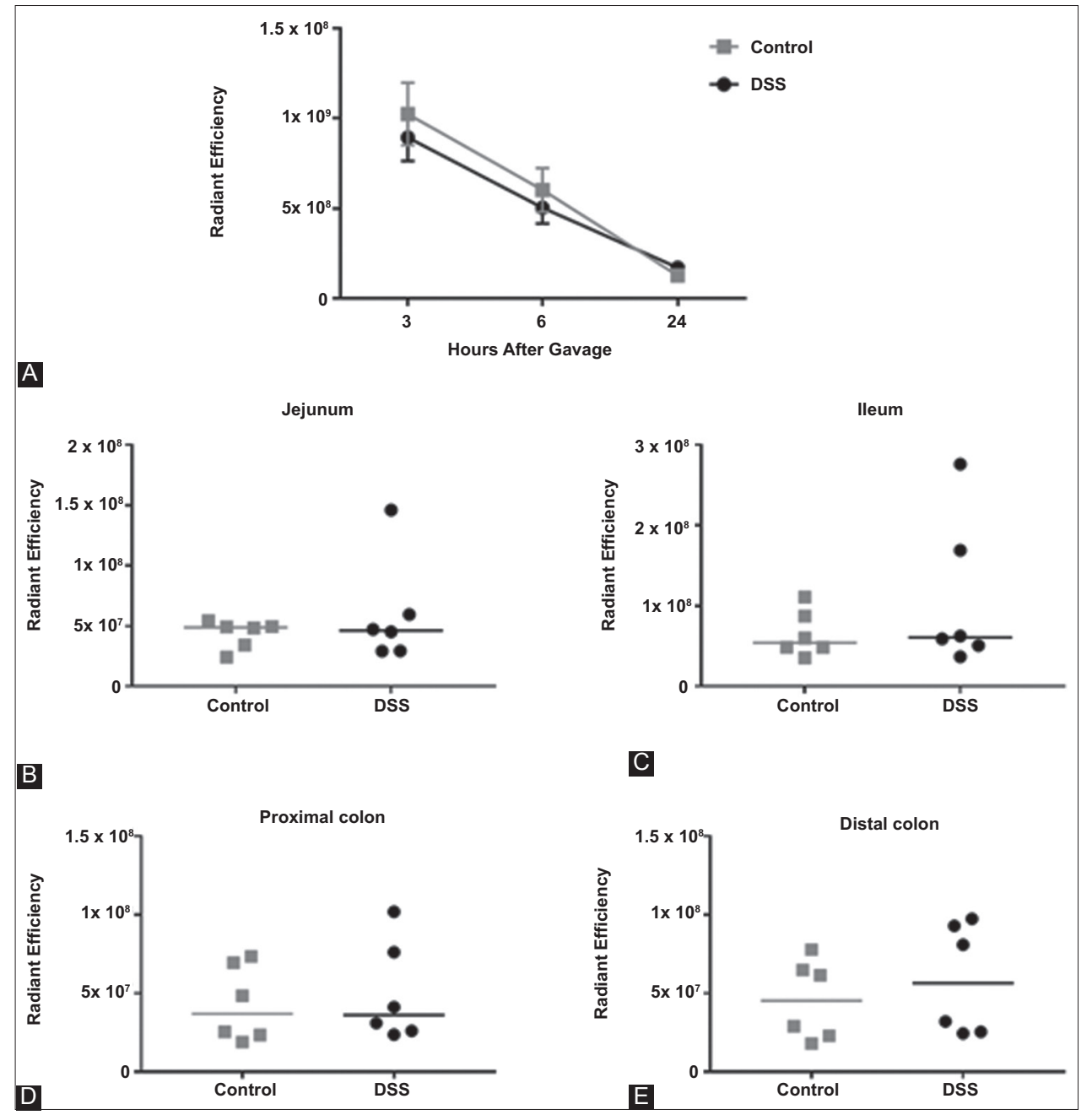

Figure 4 (A) Fluorescence quantification of overall radiant efficiency from in vivo optical images at 3, 6, and 24 h post-gavage of silica particles in control and dextran sulfate sodium (DSS)-treated rats. (B-E) Radiant efficiency from different GIT regions

Data are shown as mean $\pm S E M, P>0.05$. Lines represents medians, $P>0.05$

and induces UC by disrupting the tight junctions at the distal colon, allowing the malabsorption of water and electrolytes, as well as allowing bacterial infiltration into the submucosa, triggering inflammation [26]. Note that albino Wistar rats have a reported resistance to DSS-induced injury compared to Sprague Dawley rats; hence $4.5 \%$ DSS for 7 days was needed in the current study to induce a mild colitis compared to other studies [24].

Although the body weights of DSS-treated rats were lower compared with controls, a weight loss of $1-2 \%$ was only seen in the DSS groups from day 1 to 2 . Further, although the reparation process of the rat intestinal tract caused a minor decrease in the DAI score starting from day 8, UC was still present, as seen in the stool consistency and perianal bleeding scores, which are major indicators for UC. The DSS rats in our study had a higher peak DAI score of 6 , but a lower histopathological score of 7 compared to another study which reported lower DAI scores but higher histopathological scores (3.5 and 12, respectively) [27]. Histopathological examination of the DSS distal colon in our study showed epithelial disruption, neutrophil infiltration and submucosal thickening, whereas in Sprague Dawley rats, which are more sensitive to DSS-induced injury, crypt and goblet cell loss was also seen [21]. Nevertheless, a mild colitis is likely more reflective of clinical UC (as compared to confluent ulceration as seen in other severe models).

In this line of research it is important to ensure the fluorescent tag will not dissociate from the silica particle spontaneously or by enzyme-catalysed reactions while in vivo. The AF633 tags used in this study were covalently conjugated to the silica particles, forming a permanent bond between the AF633 and the silica particle that prevents dissociation of the tag from the particle. This ensures that the silica particles can be traced in vivo and ex vivo, with data quantifying the tagged silica particle accumulation instead of the dissociated fluorescent tags. Although we are confident the fluorescence quantified was an indication of the accumulated silica particles, we observed no significant increase in accumulation 

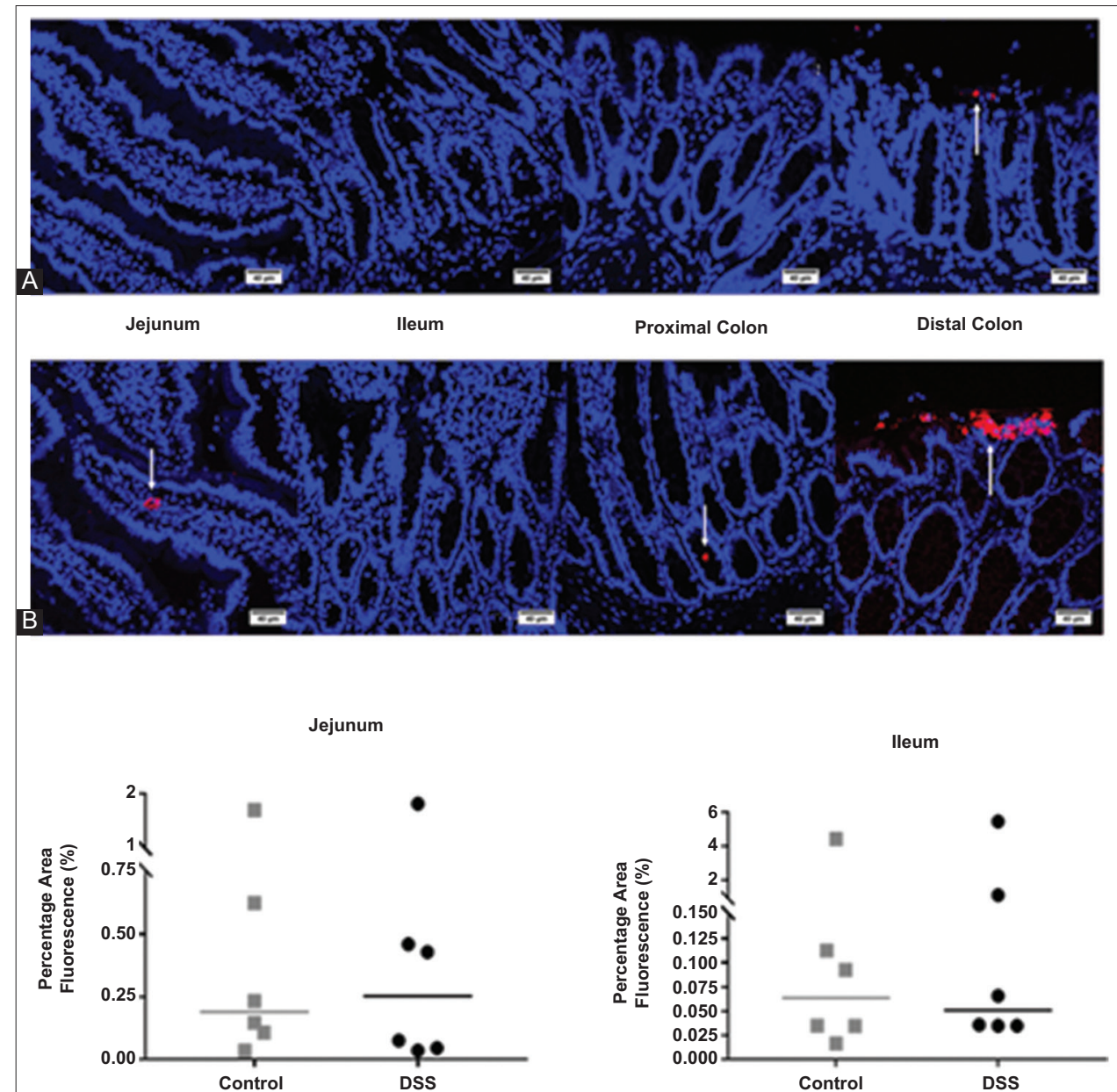

C
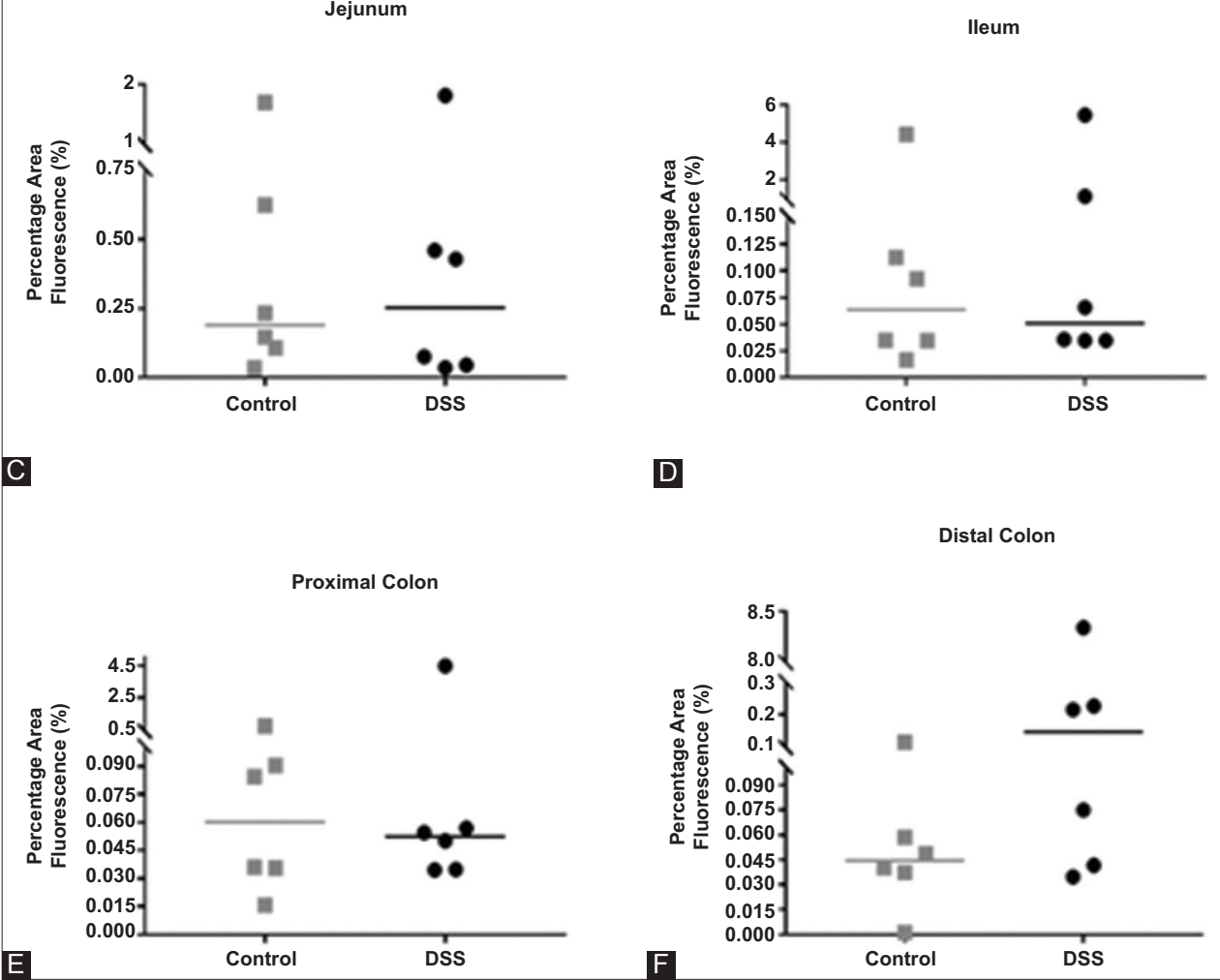

Figure 5 Representative 4',6-diamidino-2-phenylindole (DAPI) high-resolution images (400× magnification) of gastrointestinal tract (GIT) regions from (A) control and (B) dextran sulfate sodium (DSS) rats show presence of silica particles (white arrow). Blue: DAPI, red: AF633-silica particles. (C-F) Percentage area of fluorescence from different GIT regions from both groups, lines represents medians, $\mathrm{P}>0.05$

in DSS-treated compared to control rats, most probably because of the variability in fluorescence and the relatively mild inflammation induced.

Accumulation of silica particles in the stomach was expected at $3 \mathrm{~h}$; however, at $6 \mathrm{~h}$ the AF633-silica particles were expected to migrate towards the distal intestinal tract, especially in DSS-treated rats, since UC patients tend to have faster colonic transit times [28-30]. Furthermore, previous work has shown that stress causes a faster intestinal transit time in rats [29]. Therefore, it was expected that stress from the oral gavage and anesthetic would cause silica particle accumulation at the distal ileum or proximal colon at $6 \mathrm{~h}$. In addition, while it can be concluded that there was no difference in average particle accumulation between control 


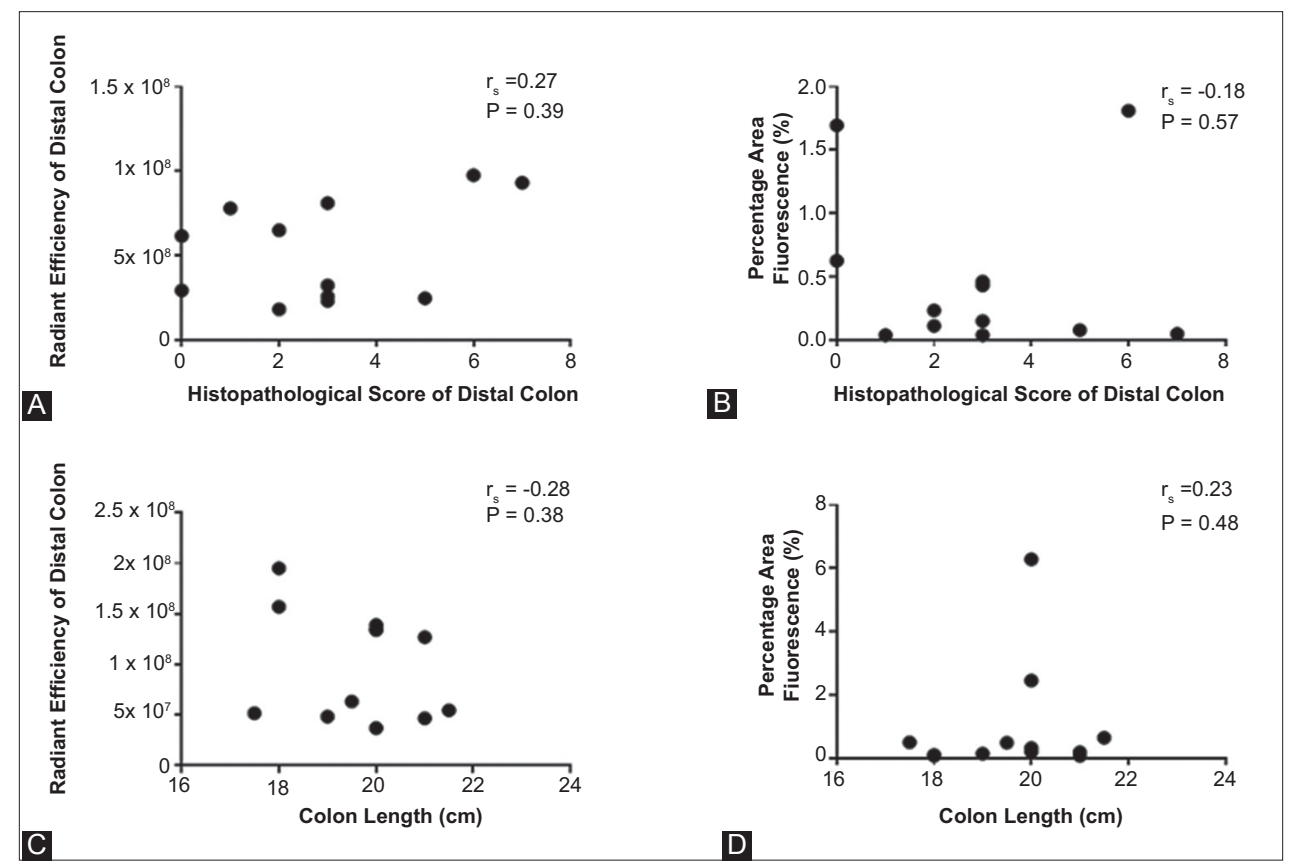

Figure 6 Correlation between rat histopathological scores of the distal colon, and (A) ex vivo radiant efficiency of the colon, and (B) percentage area of fluorescence. Correlations between rat colon lengths and (C) ex vivo radiant efficiency of the colon, and (D) percentage area of fluorescence

and DSS-treated rats in our study, these conclusions will need to be confirmed in a larger animal study. Finally, fluorescence from excreted silica particles that adhered to the skin of rats or reentered the system via coprophagy seen at $24 \mathrm{~h}$ may also have skewed the results.

The main hypothesis of this study was that more silica particles would accumulate in a more severely inflamed intestinal tract. This was demonstrated in a previous study, when accumulation of particles was plotted against colon length: a shorter colon length indicated more severe inflammation [31]. Their data showed that accumulation of particles was significantly greater with severe inflammation. In contrast, we found no significant correlation between severity of DAI and silica particle accumulation. This was most likely because of the mild inflammation induced in the colon, which may affect silica particle accumulation and absorption, causing the variability seen in silica particle accumulation in the GIT regions.

Another potential explanation of the discrepancies between the present study and previous studies is that the silica particles may be adhering to the mucus layer in the rat's intestinal tract [32]. A high turnover rate (4-6 h) of the mucus in the intestinal tract may also affect silica particle accumulation [33]. Furthermore, any silica particles trapped in the mucus would have been removed during tissue preparation before being absorbed through the GIT epithelium. Indeed, previous work in rats has shown that the accumulation of orally gavaged polystyrene fluorescent particles decreased by $38.6 \%$ when the mucus was removed from inflamed colonic tissue [32], suggesting that a large number of particles adhere to the mucus layer. Consequently, future studies are needed that optimize transport through the mucus barrier, utilizing different charge, size and surface characteristics, to enhance the uptake of silica particles across the epithelium.

In conclusion, silica particles have received significant attention concerning their potential role in protecting and delivering biologics in the body. DSS was able to successfully induce mild colitis in our albino Wistar rats. However, no correlation between DAI and silica particle accumulation was observed in this specific study. Quantifiable significance was difficult to establish given the mild nature of the colitis, the subjective nature of the scoring, the large variability of the DAI and fluorescent data, and the small sample size of the groups for a study of this nature. Despite the lack of significance, silica particles may have potential as an oral drug delivery method for UC treatment. A more exhaustive study, using these silica particles loaded with conventional UC therapeutics to target colonic inflammation, may be required to compare efficiency between silica particles and conventional UC treatment methods.

\section{Acknowledgments}

The authors would like to thank Adelaide Microscopy, University of Adelaide, for their technical expertise and assistance with the imaging and microscopy work. We also thank Matthew Teusner from Ceramisphere Pty Ltd for his expertise regarding the synthesis of the silica particles and Ceramisphere Pty Ltd for providing the silica particles. 


\section{Summary Box}

\section{What is already known:}

- Ulcerative colitis (UC) is a lifelong inflammatory bowel disease that has a significant impact on the patient's quality of life

- Conventional methods of administering UC therapeutics are suboptimal and associated with side effects

- Silica particles are a potential alternative delivery method for UC therapeutics because of their promising drug-loading and safety profiles

\section{What the new findings are:}

- Silica particles were able to accumulate in the intestinal tract

- No correlation was observed between the degree of colonic inflammation and silica particle accumulation

- Silica particles may need to be functionalized to enhance uptake across the intestinal epithelium

\section{References}

1. Wilson J, Hair C, Knight R, et al. High incidence of inflammatory bowel disease in Australia: a prospective population-based Australian incidence study. Inflamm Bowel Dis 2010;16:1550-1556.

2. Sartor RB. Mechanisms of disease: pathogenesis of Crohn's disease and ulcerative colitis. Nat Clin Pract Gastroenterol Hepatol 2006;3:390-407.

3. Guan Q, Zhang J. Recent advances: the imbalance of cytokines in the pathogenesis of inflammatory bowel disease. Mediators Inflamm 2017;2017:4810258.

4. Heller F, Florian P, Bojarski C, et al. Interleukin-13 is the key effector Th2 cytokine in ulcerative colitis that affects epithelial tight junctions, apoptosis, and cell restitution. Gastroenterology 2005; 129:550-564.

5. Eshuis EJ, Bemelman WA, Stokkers PC. Infliximab for the treatment of ulcerative colitis. Expert Rev Gastroenterol Hepatol 2009;3:219-229.

6. Garud S, Peppercorn MA. Ulcerative colitis: current treatment strategies and future prospects. Therap $A d v$ Gastroenterol 2009;2:99-108.

7. Westbrook JI, Rob MI, Woods A, Parry D. Errors in the administration of intravenous medications in hospital and the role of correct procedures and nurse experience. BMJ Qual Saf 2011;20:1027-1034.

8. Loh CS, MacRobert AJ, Bedwell J, Regula J, Krasner N, Bown SG. Oral versus intravenous administration of 5-aminolaevulinic acid for photodynamic therapy. Br J Cancer 1993;68:41-51.

9. Powell-Tuck J, Bown RL, Lennard-Jones JE. A comparison of oral prednisolone given as single or multiple daily doses for active proctocolitis. Scand J Gastroenterol 1978;13:833-837.

10. Harter JG, Reddy WJ, Thorn GW. Studies on an intermittent corticosteroid dosage regimen. $N$ Engl J Med 1963;269:591-596.

11. Mahadevan U. Medical treatment of ulcerative colitis. Clin Colon Rectal Surg 2004;17:7-19.
12. Barbé C, Bartlett J, Kong L, et al. Silica particles: a novel drugdelivery system. Adv Mater 2004;16:1959-1966.

13. Barbé C, Kong L, Finnie K, et al. Sol-gel matrices for controlled release: from macro to nano using emulsion polymerisation. J SolGel Sci Technol 2008;46:393-409.

14. Watermann A, Brieger J. Mesoporous silica nanoparticles as drug delivery vehicles in cancer. Nanomaterials 2017;7:189.

15. Zhang M, Xu C, Liu D, Han MK, Wang L, Merlin D. Oral delivery of nanoparticles loaded with ginger active compound, 6-shogaol, attenuates ulcerative colitis and promotes wound healing in a murine model of ulcerative colitis. J Crohns Colitis 2018;12:217-229.

16. Yoshida T, Yoshioka Y, Takahashi $\mathrm{H}$, et al. Intestinal absorption and biological effects of orally administered amorphous silica particles. Nanoscale Res Lett 2014;9:532.

17. Barbé CJ, Bartlett JR. Controlled release ceramic particles, compositions thereof, processes of preparation and methods of use. WO 01/62232 Granted in Europe, US, Mexico, NZ, Australia, India, Canada and Japan.

18. National Health and Medical Research Council. Australian code of practice for the care and use of animals for scientific purposes. Canberra: National Health and Medical Research Council, 2013.

19. Napierska D, Thomassen LC, Rabolli V, et al. Size-dependent cytotoxicity of monodisperse silica nanoparticles in human endothelial cells. Small 2009;5:846-853.

20. Dai C, Zheng CQ, Meng FJ, Zhou Z, Sang LX, Jiang M. VSL\#3 probiotics exerts the anti-inflammatory activity via PI3k/Akt and NF- $\kappa B$ pathway in rat model of DSS-induced colitis. Mol Cell Biochem 2013;374:1-11.

21. Howarth GS, Xian CJ, Read LC. Insulin-like growth factor-I partially attenuates colonic damage in rats with experimental colitis induced by oral dextran sulphate sodium. Scand J Gastroenterol 1998;33:180-190.

22. Schindelin J, Rueden CT, Hiner MC, Eliceiri KW. The ImageJ ecosystem: an open platform for biomedical image analysis. $\mathrm{Mol}$ Reprod Dev 2015;82:518-529.

23. Philip AK, Philip B. Colon targeted drug delivery systems: a review on primary and novel approaches. Oman Med J 2010;25:79-87.

24. Gaudio E, Taddei G, Vetuschi A, et al. Dextran sulfate sodium (DSS) colitis in rats: clinical, structural, and ultrastructural aspects. Dig Dis Sci 1999;44:1458-1475.

25. Chassaing B, Aitken JD, Malleshappa M, Vijay-Kumar M. Dextran sulfate sodium (DSS)-induced colitis in mice. Curr Protoc Immunol 2014;104:Unit 15.25.

26. Poritz LS, Garver KI, Green C, Fitzpatrick L, Ruggiero F, Koltun WA. Loss of the tight junction protein ZO-1 in dextran sulfate sodium induced colitis. J Surg Res 2007;140:12-19.

27. Atkins HL, Geier MS, Prisciandaro LD, et al. Effects of a Lactobacillus reuteri $\mathrm{BR} 11$ mutant deficient in the cystine-transport system in a rat model of inflammatory bowel disease. Dig Dis Sci 2012;57:713-719.

28. Hebden JM, Blackshaw PE, Perkins AC, Wilson CG, Spiller RC. Limited exposure of the healthy distal colon to orally-dosed formulation is further exaggerated in active left-sided ulcerative colitis. Aliment Pharmacol Ther 2000;14:155-161.

29. Enck P, Merlin V, Erckenbrecht JF, Wienbeck M. Stress effects on gastrointestinal transit in the rat. Gut 1989;30:455-459.

30. Tomlin J, Brown N, Ellis A, Carlsson A, Bogentoft C, Read NW. The effect of liquid fibre on gastric emptying in the rat and humans and the distribution of small intestinal contents in the rat. Gut 1993;34:1177-1181.

31. Watanabe A, Tanaka H, Sakurai Y, et al. Effect of particle size on their accumulation in an inflammatory lesion in a dextran sulfate sodium (DSS)-induced colitis model. Int J Pharm 2016;509:118-122.

32. Lamprecht A, Schäfer U, Lehr CM. Size-dependent bioadhesion of micro- and nanoparticulate carriers to the inflamed colonic mucosa. Pharm Res 2001;18:788-793.

33. Lehr C-M, Poelma FGJ, Junginger HE, Tukker JJ. An estimate of turnover time of intestinal mucus gel layer in the rat in situ loop. Int J Pharm 1991;70:235-240. 